On the Growth of the Number of Bound States with Increase in Potential Strength

Barry Simon

Citation: Journal of Mathematical Physics 10, 1123 (1969); doi: 10.1063/1.1664948

View online: http://dx.doi.org/10.1063/1.1664948

View Table of Contents: http://aip.scitation.org/toc/jmp/10/7

Published by the American Institute of Physics

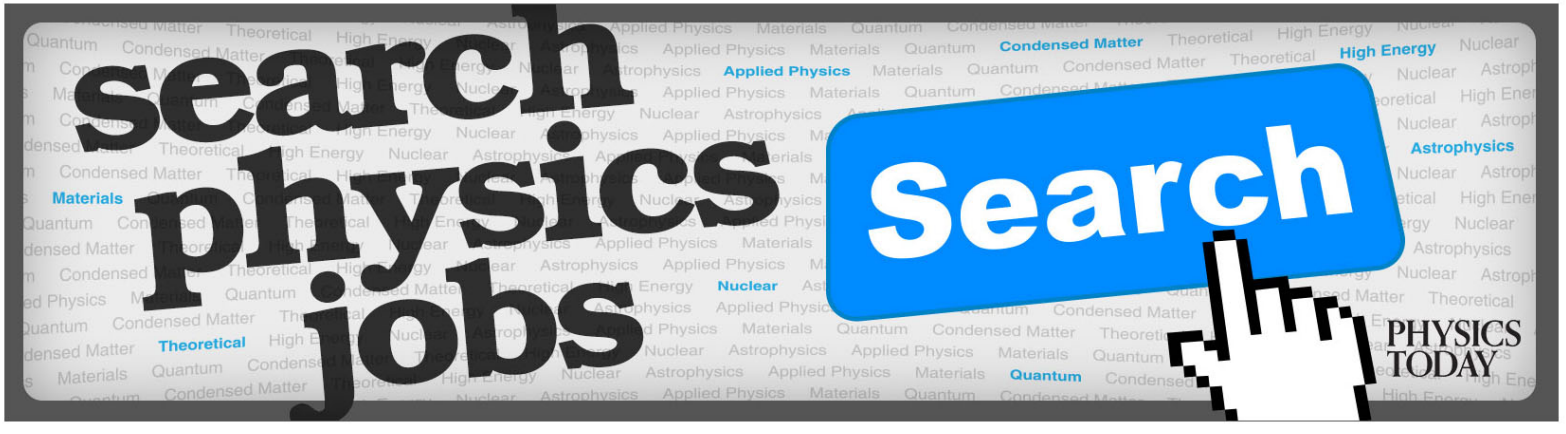




\title{
On the Growth of the Number of Bound States with Increase in Potential Strength*
}

\author{
BARRY SIMON† \\ Princeton University, Princeton, New Jersey
}

(Received 21 November 1968)

\begin{abstract}
For a wide class of potentials, it is shown that $N(\lambda)$, the number of bound states (including multiplicity) of $-\Delta+\lambda V$, obeys the conditions

$$
A \lambda^{\frac{3}{2}}<N(\lambda)<B \lambda^{\frac{3}{2}}
$$
\end{abstract}

for $\lambda$ sufficiently large. $A$ and $B$ are positive finite numbers. In the centrally symmetric cases, a related growth condition on $l_{\max }(\lambda)$, the largest $l$ channel with bound states, is also obtained, namely,

$$
a \lambda^{\frac{1}{2}}<l_{\max }(\lambda)<b \lambda^{\frac{1}{2}} .
$$

Finally, we discuss analogous results for a larger class of central potentials and for the many-body case.

\section{INTRODUCTION}

There are a fairly large number of results giving both lower and upper bounds on the number of bound states in a given $l$ channel for a central potential. ${ }^{1-8}$ From these, limits can be developed on the growth of the number of states in a fixed channel as the strength of the potential increases. The strongest general result of this nature has been obtained by Calogero. ${ }^{4}$ If $n_{l}(\lambda V)$ is the number of bound states (not counting multiplicity) of angular momentum $l$ for the operator $-\Delta+\lambda V$, then Calogero shows that

$$
C \lambda^{\frac{1}{2}}<n_{l}(\lambda V)<D \lambda^{\frac{1}{2}}
$$

for $\lambda$ sufficiently large ( $\lambda$ will always be positive in this paper) and for a large class of potentials. $C$ and $D$ are, of course, $V$ - and $l$-dependent (actually, $D$ can be chosen independently of $l$ ).

For a restricted class of potentials (negative nonincreasing as $r \rightarrow 0$ ), Chadan ${ }^{6}$ has shown that

$$
\lim _{\lambda \rightarrow \infty} n_{l}(\lambda V) / \lambda^{\frac{1}{2}}
$$

exists and has a simple form in terms of $V$.

For some reason, there seem to be almost no results on the growth of the total number of bound

\footnotetext{
* This research partially sponsored under Air Force Research and Development Command contract AF49(638)1545.

+ National Science Foundation pre-doctoral fellow.

1 V. Bargmann, Proc. Natl. Acad. Sci. U.S. 38, 961 (1952).

${ }^{2}$ F. Calogero, Nuovo Cimento 36, 199 (1965).

${ }^{3}$ J. Schwinger, Proc. Natl. Acad. Sci. U.S. 47, 122 (1961); F. Calogero, J. Math. Phys. 6, 161 (1965).

${ }^{4}$ F. Calogero, Commun. Math. Phys. 1, 80 (1965).

5 F. Calogero, J. Math. Phys. 6, 1105 (1965); W. Frank, ibid. 8, 466, (1967); G. Ghirardi and A. Rimini, ibid. 6, 40 (1965).

B K. Chadan, Nuovo Cimento 58A, 191 (1968).

7 J. H. E. Cohn, J. London Math. Soc. 40, 523 (1965); 41, 474 (1966).

${ }^{8} \mathrm{~A}$ complete and coherent presentation of Calogero's results (Refs. 2-5) can be found in F. Calogero, Variable Phase Approach to Potential Scattering (Academic Press Inc., New York, 1967).
}

states (counting multiplicity)

$$
N(V)=\sum_{l=0}^{\infty}(2 l+1) n_{l}(V) .
$$

In this paper we show that, for a large class of not necessarily central potentials, there are nonzero constants $A$ and $B$ such that, for sufficiently large $\lambda$ (Theorems 2, 3, and 6),

$$
A \lambda^{\frac{3}{2}}<N(\lambda V)<B \lambda^{\frac{3}{2}} \text {. }
$$

We also show (Theorems 1 and 5) that the number of angular-momentum channels with bound states goes as $\lambda \frac{1}{2}$.

The conditions we impose on centrally symmetric $V$ are the following:

(A) For all $\lambda,-\Delta+\lambda V$ has no eigenvalues of positive energy and the negative-energy spectrum is purely discrete of finite multiplicity.

(B) $I(V) \equiv \int_{0}^{\infty} d r \quad r|V(r)|<\infty$.

(C) $\inf \left[r^{2} V(r)\right] \equiv-L>-\infty$.

(D) For some $\alpha>0,\{r \mid V(r)<-\alpha\}$ has a nonempty interior.

The characterization of the negative spectrum in (A) can be assured by very weak conditions. ${ }^{9}$ The absence of positive-energy bound states is assured by fairly mild conditions. ${ }^{10}$

(B) is the standard condition of Jost and Pais. ${ }^{11}$ It can be replaced by the alternate condition:

$\left(\mathrm{B}^{\prime}\right) V$ is minorized by a monotonically increasing potential $\tilde{V}$ with

$$
Q(\tilde{V})=\int_{0}^{\infty} d r|\tilde{V}(r)|^{\frac{1}{2}}<\infty .
$$

T. Kato, Progr. Theoret. Phys. (Kyoto) Suppl. 40, 3 (1967); R. Courant and D. Hilbert, Methods of Mathematical Physics (Interscience Publishers. Inc.. New York, 1953), Vol. I, p. 448.

${ }_{10}$ T. Kato, Commun. Pure Appl. Math. 12, 403 (1959); F. Odeh, Proc. Am. Math. Soc.' 16, 363; B. Simon, Commun. Pure Appl. Math. (to be published); J. Weidmann, Math. Z. 98, 268 (1967). ${ }_{11}$ R. Jost and A. Pais, Phys. Rev. 82, 840 (1951). 
We prove our results initially in the centrally symmetric case and discuss the easy extensions to the noncentral case in Sec. V.

\section{THE UPPER BOUND}

First we remark that there is an upper bound weaker than ours implicit in Bargmann's result ${ }^{1}$ that

$$
n_{l}(W)<I(W) / 2 l+1
$$

for any potential $W$. For then $n_{l}(W)=0$, if $2 l+1>$ $I(W)$, so that

$$
\begin{aligned}
N(W) & <\sum_{l=0}^{[(I-1) / 2]}(2 l+1) n_{l}(W) \\
& <I\left\{1+\left[\frac{1}{2}(I-1)\right]\right\}<\frac{1}{2} I(I+1) .
\end{aligned}
$$

For $W=\lambda V, I(W)=\lambda I(V)$, so we see that

$$
N(\lambda V)<\frac{1}{2} \lambda I(\lambda I+1)<I^{2} \lambda^{2}, \text { for } \lambda>(I)^{-1} .
$$

Our stronger result is obtained by a better estimate of the maximum $l$-channel with a bound state in it; we designate this $l$-value $l_{\max }(\lambda)$.

Theorem 1: If (A) and (C) hold, then for all $\lambda$,

$$
l_{\max }(\lambda)<(L)^{\frac{1}{2}} \lambda^{\frac{1}{2}} \text {. }
$$

Proof ${ }^{12}$ : Since $-d^{2} / d r^{2}$ is a positive operator and $-\Delta+\lambda V$ has no positive eigenvalues, there are no bound states in the $l$ channel, if

$$
l(l+1) / r^{2}+\lambda V(r)>0, \text { for all } r .
$$

But

$$
l(l+1) / r^{2}+\lambda V>r^{-2}[l(l+1)-\lambda L],
$$

so $n_{l}(\lambda V)=0$, if $l(l+1)>\lambda L$; i.e., if

$l>(\lambda L)^{\frac{1}{2}}$, then $n_{l}(\lambda V)=0$, i.e., $l_{\max }(\lambda)<(\lambda L)^{\frac{1}{2}}$.

Theorem 2: If (A), (B), and (C) hold, then, for all $\lambda>L^{-1}$,

$$
N(\lambda V)<\left[2 L^{\frac{1}{2}} I\right] \lambda^{\frac{3}{2}} .
$$

Proof: By Bargmann's condition (2),

$$
(2 l+1) n_{l}(\lambda V)<\lambda I
$$

so

$$
\begin{aligned}
N(\lambda V) & <\sum_{l=0}^{l_{\max }}(2 l+1) n_{l}(\lambda V)<(\lambda I)\left(l_{\max }+1\right) \\
& <(\lambda I)\left(\lambda^{\frac{1}{2}} L^{\frac{1}{2}}+1\right)<2 \lambda^{\frac{3}{2}} I L^{\frac{1}{2}}
\end{aligned}
$$

if $\lambda L>1$.

If $\left(B^{\prime}\right)$ holds instead of (B), we replace Bargmann's bound (2) with that of Calogero ${ }^{2}$ (see also Ref. 7):

$$
n_{l}(V)<(2 / \pi) Q(\tilde{V}), \text { for all } l .
$$

${ }^{12}$ An alternate proof can be based on the bound given in $F$. Calogero and G. Cosenza, Nuovo Cimento 45, 867 (1966).
Theorem 3: If (A), $\left(\mathrm{B}^{\prime}\right)$, and $(\mathrm{C})$ hold, then for all $\lambda>L^{-1}$,

$$
N(\lambda V)<(8 / \pi) L Q(\tilde{V}) \lambda^{\frac{3}{2}} .
$$

Proof: From Calogero's condition,

so that

$$
n_{l}(\lambda V) \leq \lambda^{\frac{1}{2}}[(2 / \pi) Q(\tilde{V})]
$$

if $\lambda L \geq 1$.

$$
\begin{aligned}
N(\lambda V) & \leq \lambda^{\frac{1}{2}}\left[\frac{2}{\pi} Q(\tilde{V})\right] \sum_{l=0}^{l_{\max }}(2 l+1) \\
& =\lambda^{\frac{1}{2}}[(2 / \pi) Q(\tilde{V})]\left(l_{\max }+1\right)^{2} \\
& \leq \lambda^{\frac{3}{2}}[(8 / \pi) L Q(\tilde{V})],
\end{aligned}
$$

\section{A STRONG RAYLEIGH-RITZ PRINCIPLE}

The nub of the proofs of the lower bounds is a form of the Rayleigh-Ritz principle which is more explicit than is usually found. While Theorem 4 is no doubt well known, its value for proving the existence of bound states does not seem to have been fully appreciated. It is essentially the principle used by Kato in his proof that the helium Hamiltonian has at least 25585 bound states. ${ }^{13}$

Theorem 4: Let $H$ be a self-adjoint operator on a domain $D$ and let

where

$$
\mu_{n}=\sup _{\Phi_{1}, \cdots, \Phi_{n-1}}\left\{\inf _{\Psi \in U\left(\Phi_{1}, \cdots, \Phi_{n-1}\right)}(\Psi, H \Psi)\right\},
$$

$$
\begin{aligned}
U\left(\Phi_{1}, \cdots,\right. & \left.\Phi_{n-1}\right) \\
& =\left\{\Psi \mid \Psi \in D,\|\Psi\|=1 \text { and }\left(\Phi_{i}, \Psi\right)=0\right\} .
\end{aligned}
$$

Then for each fixed $n$, either

(a) $\mu_{n}$ is the $n$th eigenvalue counting multiplicity or

(b) $\mu_{n}$ is the bottom of the essential spectrum and $\mu_{n}=\mu_{n+1}=\mu_{n+2}=\cdots$.

Moreover, there are at most $n-1$ eigenvalues less than $\mu_{n}$. (The essential spectrum is the set of points in the spectrum which are not isolated points of finite multiplicity.) The theorem holds if we replace $D$ in the definition of $U$, by $D_{0}$, the domain of $H$ as a bilinear form, i.e., the domain of $|H|^{\frac{1}{2}}$ as an operator.

We do not write out a proof of this theorem, as it is completely straightforward if one is willing to use a little spectral theory. The power of Theorem 4 comes when it is combined with condition (A); for if $\mu_{n}$ is negative and (A) holds, then (b) cannot be true and so (a) must hold. We remark that in application, $H$ is either $-\Delta+V$ or $-\Delta+V$ restricted to an angularmomentum subspace.

\footnotetext{
${ }^{13}$ T. Kato, Trans. Am. Math. Soc. 70, 212 (1951).
} 
We first prove some corollaries which we need.

Corollary 1: If (D) holds, then $-\Delta+\lambda V$ has a bound $p$-state for $\lambda$ sufficiently large.

Proof: Pick a smooth function $\phi$ of $r$ of compact support, so that support of $\phi$ is contained in the set with $V(r)<0$. Let $\Psi^{\prime}(r, \theta, \phi)=r \phi(r) Y_{1}^{0}(\theta, \phi)$. Then $(\Psi, V \Psi)<0$ and so, for $\lambda$ sufficiently large, $-\left(\Psi^{\prime}, \Delta \Psi^{\circ}\right)+\lambda(\Psi, V \Psi)<0$, i.e., $-\Delta+\lambda V$ has a bound $p$-state.

Corollary $2: n_{l}(\lambda V)$ is a monotonically increasing function of $\lambda$.

\section{Proof:}

$$
-\Delta+\lambda_{0} W=\lambda_{0}(-\Delta+W)+\left(1-\lambda_{0}\right)(-\Delta) .
$$

For $\lambda_{0}>1,\left(1-\lambda_{0}\right)(-\Delta)$ is a negative operator, so that by Theorem 4 applied to the operators on the space of functions of angular momentum $l, n_{l}\left(\lambda_{0} W\right) \geq$ $n_{l}(W)$, for all $W$. Letting $W=\lambda V$ and $\lambda_{0} \lambda=\lambda_{1}$, we see that $n_{l}\left(\lambda_{1} V\right) \geq n_{l}(\lambda V)$ if $\lambda_{1}>\lambda$.

Corollary 3: For any central potential $V$, and for $l \geq 1$,

$$
n_{l}\left[\frac{1}{2} l(l+1) V\right] \geq n_{1}(V) .
$$

Proof:

$$
\begin{aligned}
-\frac{d}{d r^{2}}+ & \frac{l(l+1)}{r^{2}}+\frac{l(l+1)}{2} V \\
=\frac{l(l+1)}{2} & \left\{-\frac{d^{2}}{d r^{2}}+\frac{2}{r^{2}}+V\right\} \\
& +\frac{2-l(l+1)}{2}\left(-\frac{d^{2}}{d r^{2}}\right),
\end{aligned}
$$

where

$$
\frac{2-l(l+1)}{2}\left(-\frac{d^{2}}{d r^{2}}\right)
$$

is negative, so that the left-hand side of (4) has at least as many bound states as the right-hand side, i.e.,

$$
n_{l}\left[\frac{1}{2} l(l+1) V\right] \geq n_{1}(V) .
$$

Corollary 4: Let $V_{1}$ obey condition (A) and let $V_{2}$ by any potential with $V_{2}(r) \geq V_{1}(r)$ for all $r$. If all the negative-energy eigenfunctions of $-\Delta+V_{2}$ are in the domain of $-\Delta+V_{1}$ as a bilinear form, then $N\left(\lambda V_{1}\right)$ is at least as large as the number of negative-energy eigenvalues of $\lambda V_{2}$.

The proof is trivial; however, we remark that careful applications should not ignore the domain con- dition. We do not have a pathological $V_{2}$ in mind when we distinguish the negative-energy eigenvalues; rather we will not require $V_{2}$ to go to 0 at $\infty$ and, in fact, will take $V_{2} \rightarrow \infty$ as $\mathbf{r} \rightarrow \infty$.

\section{THE LOWER BOUNDS}

Theorem 5: Let $V$ obey (A) and (D) and suppose $n_{1}\left(\lambda_{0} V\right) \geq 1$. Then, for $\lambda>\lambda_{0}$,

$$
l_{\max }(\lambda) \geq\left(\frac{1}{2} \lambda_{0}^{-\frac{1}{2}}\right) \lambda^{\frac{1}{2}} .
$$

[Note: By Corollary 1,(D) implies that some $\lambda_{0}$ exists.]

Proof: By Corollary 3,

$$
n_{l}\left[\frac{1}{2} \lambda_{0} l(l+1) V\right] \geq 1 .
$$

Thus, if $\lambda \geq \frac{1}{2} \lambda_{0} l(l+1), n_{l}(\lambda V) \geq 1$ (by Corollary 2 ). Thus, if $l \leq\left(\lambda / \lambda_{0}\right)^{\frac{1}{2}}$ and $l \geq 1, n_{l}(\lambda V) \geq 1$; i.e.,

$$
l_{\max }(\lambda) \geq\left[\left(\frac{\lambda}{\lambda_{0}}\right)^{\frac{1}{2}}\right] \geq \frac{1}{2}\left(\frac{\lambda}{\lambda_{0}}\right)^{\frac{1}{2}}, \quad \text { if } \quad \lambda \geq \lambda_{0} .
$$

Corollary 5: If $V$ obeys conditions (A) and (D), then, for all $\lambda \geq \lambda_{0}\left(\lambda_{0}\right.$ as in Theorem 5),

Proof:

$$
N(\lambda V)>\lambda / \lambda_{0} \text {. }
$$

$$
\begin{aligned}
N(\lambda) & >\sum_{l=0}^{l_{\max }}(2 l+1)=\left(l_{\max }+1\right)^{2} \\
& \geq\left\{\left[\left(\lambda / \lambda_{0}\right)^{\frac{1}{2}}\right]+1\right\}^{2} \geq \lambda / \lambda_{0} . \quad \text { Q.E.D. }
\end{aligned}
$$

To get an improvement on the growth rate of Corollary 5, a comparison with specific potentials seems necessary. A comparison proof is also possible for obtaining the upper bounds.

Lemma 1: Let $D$ be the region of $\mathbb{R}^{3}$ with

$$
\left|x-x_{0}\right|<L, \quad\left|y-y_{0}\right|<L, \quad\left|Z-Z_{0}\right|<L,
$$

and let

$$
V_{0}(r)=\left\{\begin{array}{ll}
-P, & x \in D, \\
\infty, & x \in D,
\end{array} \text { with } P>0\right.
$$

Let $N\left(\lambda V_{0}\right)$ be the number of bound states of negative energy for $-\Delta+\lambda V_{0}$. Then for $\lambda$ sufficiently large,

$$
N\left(\lambda V_{0}\right)>A \lambda^{\frac{3}{2}} \text {. }
$$

Proof: The eigenvalues of $-\Delta+\lambda V_{0}$ are

$$
E_{\mathrm{n}}(\lambda)=(\pi / 2 L)^{2}\left(n_{1}^{2}+n_{2}^{2}+n_{3}^{2}\right)-\lambda P,
$$

where $n_{1}, n_{2}, n_{3}$ are positive integers. Thus, $N\left(\lambda V_{0}\right)$ asymptotically approaches the volume of an octant of a sphere of radius $C \lambda^{\frac{1}{2}}$. As a result, $N\left(\lambda V_{0}\right) / \lambda^{\frac{3}{2}}$ actually approaches a limit which is positive. 
Theorem 6: Let $V$ obey (C) and (D) and suppose $D\left(H_{0}+\lambda V\right) \supset D\left(H_{0}\right) \cap D(V)$, where $D(X)$ is the domain of $X$ as a bilinear form. Then, for $\lambda$ sufficiently large:

$$
N(\lambda V) \geq A \lambda^{\frac{3}{2}}
$$

Proof: Pick a ball $B$ in $\mathbb{R}^{3}$ so that $V(\mathbf{r})<-P$ in $B$. Inside $B$, find a region $D$ as in Lemma 1 and let $V_{0}$ be as in that lemma. The eigenfunctions for the square well are in $D(V)$ by $(\mathrm{C})$ and they are in $D\left(H_{0}\right)$ [they are not in the domain of $H_{0}$ as an operator; to be in $D\left(H_{0}\right)$, they need only possess $L^{2}$ first derivatives]; thus, from the domain condition, Corollary 4 , and Lemma 1 , the theorem follows.

Q.E.D.

We remark first that condition (C) is much stronger than what we need. It is sufficient that $V$ be negative in some ball $B$ for which

$$
\int_{B}|V(\mathbf{r})|^{2} d^{3} r<\infty
$$

We also remark that it is almost inconceivable that one can make sense out of $H_{0}+V$ without having the domain condition hold. Examples of classes of $V$ for which it must hold are:

(1) $V \in L^{2}+L^{\infty}$ (Kato potentials);

(2) $V$ bounded below, $H_{0}+\lambda V$ defined by the Friedrichs' extension method;

(3) $V \in L^{\infty}+L^{\frac{3}{2}}$, in which case one can show that $H_{0}+\lambda V$ is bounded below and so Friedrichs' extension can be used.

\section{EXTENSIONS TO MORE GENERAL CASES}

To $N$ dimensions: It is a little enlightening to note that in $N$ dimensions $\lambda^{\frac{3}{2}}$ is replaced by $\lambda^{N / 2}$; for example, our comparison potential, the harmonic oscillator, has $N\left(\lambda V_{0}\right) \sim \lambda^{N / 2}$. Thus, we can understand the fact $n_{l}(\lambda V) \sim \lambda^{\frac{1}{2}}$ by realizing that the single-channel Schrödinger equation is essentially 1-dimensional.

To noncentral potentials: The proof of Theorem 6 carries over without change to the noncentral case. To obtain an upper bound, we need only a simple comparison potential. Let

$$
V_{\min }(r)=\min _{|\mathbf{r}|=r} V(\mathbf{r})
$$

Then, since $V_{\min }<V$, we have $N\left(\lambda V_{\min }\right)>N(\lambda V)$. If $V_{\min }$ obeys (A), (B), and (C), the upper bound given by Theorem 2 yields an upper bound for $N(\lambda V)$.

To the many-body case: As with most problems in nonrelativistic potential theory, things really get interesting in the many-body case. Also as with most problems, the two-body methods are not capable of extension. In this case, there are negative-energy continua (due to relative motion of bound clusters) which complicate the analysis and invalidate all the arguments we have used in the two-body case.

\section{ACKNOWLEDGMENTS}

It is a pleasure to thank Professor V. Bargmann and Professor A. S. Wightman for their interest and perceptive comments. 